\title{
Measurement of friction force in a toothbrush tuft using Levitation Mass Method (LMM)
}

\author{
*Joey S. Pastoril ${ }^{1}$, Kazuhide Watanabe ${ }^{2}$, Kyohei Irisa ${ }^{2}$, Irfa Aji Prayogi ${ }^{2}$ \\ Akihiro Takita ${ }^{2}$, Edwin Carcasona ${ }^{1}$ and Yusaku Fujiii ${ }^{2}$ \\ ${ }^{1}$ Department of Mechanical Engineering, University of San Carlos, Cebu City 230-0100, Philippines \\ ${ }^{2}$ School of Science and Technology, Gunma University, Kiryu Gunma 376-8515, Japan
}

*Corresponding author: jpastoril@yahoo.com

\begin{abstract}
The friction force in a single toothbrush tuft (group of bristles) is measured using Levitation Mass Method (LMM). The experimental measurement is performed by sliding a rounded tip extension block over the surface of the tuft. This extension block is connected to one end of the moving part that is a slide of pneumatic linear bearing. Since the friction of the moving part is so small that it can be neglected, the inertial force of the moving part is equal to the dynamic surface friction force of the tuft opposite to the direction of motion. The tested toothbrush tuft used is made of nylon material with 25 bristles, has a length of 10 $\mathrm{mm}$ and a diameter of $0.2 \mathrm{~mm}$. The velocity of the moving part is measured precisely from the beat frequency and the rest frequency using an optical interferometer. After getting the velocity, the other data like the acceleration, the inertial force, and the position of the mass is calculated numerically. The friction inside the bearing is determined using the same instrumentation with an average value of $2.05 \mathrm{mN}$.
\end{abstract}

Keyword: Inertial force, Dynamic force, Levitation mass method, Toothbrush tuft, Bristle

\section{Introduction}

Toothbrush is an instrument for cleaning the teeth, the tongue, and the gums of a person. The origin of toothbrush can be traced back to China about 1000 AD [1]. Typically, the toothbrush is made of soft nylon bristle with different bristle configurations that form different tuft design and arrangement. This different in configuration of bristles formed the tuft arrange at different angles (criss-cross) and at different lengths [2]. A tuft of a toothbrush is a group of bristles, which is lumped together and equally distributed over the toothbrush head. According to Fradsen [3], manual toothbrush bristles are normally end-rounded tips with a dimension of approximately $10 \mathrm{~mm}$ in length and $0.2 \mathrm{~mm}$ in diameter. Some literatures like Hotta et al. [4] talk about the plaque removal efficacy of the toothbrush has something to do with the proper bristle arrangement and material type. This is further supported by the study of Yankell [5] et al. that feathered bristles removed more plaque than end-rounded bristle.

The bristle of a toothbrush can be grouped into two major types hard and soft. Hard bristle toothbrush according to Kinoshita [6] is more effective in plaque removal than the soft bristle. The study of Beke [7] also suggested that the stiffness of bristle vary for every toothbrush. In one of his review, he said that stiffness is proportional to the square of the bristle diameter and inversely proportional to the square of the bristle length. Some literatures also mention about the optimum force required in removing the plaque during manual and power tooth brushing is about 3 to $4 \mathrm{~N}[8,9]$. However, none of them mention about how the friction force in a single toothbrush tuft (group of bristles) behaves during dynamic loading. The idea of knowing this will be an initial step to understand better on how a friction force is related to the efficacy of plaque removal during brushing of teeth.

Now since most of the method used in measuring the forces in toothbrushing, and toothbrush bristles stiffness experiments are static and the dynamic force measurement is still a developing technique in force measurement. The authors would like to apply a very accurate measurement method of the dynamic force developed by Fujii [10] to the measurement of friction force a toothbrush tuft. His method is already tested in many experiments like dynamic calibration of force transducer, micro force measurement, impact force measurement, friction force measurement and coefficient of friction measurement [11-14]. This method is called the Levitation Mass Method (LMM). The principle behind of this method is that the inertial force of a mass is levitated using a pneumatic linear bearing. Then the inertial force of the levitated mass is used as a reference force applied to the body or material under test [15]. The inertial force of the levitated mass is accurately measured using an optical interferometer [16]. 


\section{Experimental setup}

Figure 1 shows the schematic diagram of the experimental setup for the determination of friction force of the toothbrush tuft. In this experiment, the surface of the toothbrush tuft is rubbed by a rounded tip extension block connected to the linear bearing. The reference force acting on the toothbrush tuft is the inertial force of the moving mass in a linear bearing. The moving mass is set up in such a way that its linear motion is acted by minimal friction. To achieve this a pneumatic linear bearing is used wherein the moving part is levitated by the compressed air inside the bearing. During the test, the friction force is generated in the material by sliding the moving mass over the toothbrush tuft surface. The initial velocity of the moving part is manually given by tapping it.

The moving part is made from aluminum with a shape of a square pole. One of its ends is set up for an interferometer where a cube-corner prism (CC) is attached while on the other ends is where the rounded tip extension block is placed. The inertial force acting on the mass is measured precisely using an optical interferometer. The total mass $M$ of the moving part is measured precisely using an electric balance, and it is approximately equal to $31.11 \mathrm{~g}$. The material under test (toothbrush tuft) is attached to a movable holder that is placed on a fixed base. Figure 2 shows the photograph of the test section of the experimental setup and the photograph of the toothbrush tufts and bristle. The total force $F_{\text {mass }}$ acting on the moving part of the linear bearing is the product of its mass $M$ and its acceleration $a$ shown in equation 1 .

$$
F_{\text {mass }}=M a
$$

The total force $F_{\text {mass }}$ acting on the moving part is divided into two parts. The first part is the friction force $F_{\mathrm{F}}$ resulted from deformations of the softer material (tuft) to the harder material (extension block) [17]. The second part is the force acting on the linear bearing $F_{\text {bearing. So that }}$ equation 1 becomes

$$
F_{\text {mass }}=F_{\mathrm{F}}+F_{\text {bearing }}
$$

Now, if the force acting on the linear bearing $F_{\text {bearing }}$ can be neglected then by second law of newton, the friction force $F_{\mathrm{F}}$ acting opposite to the direction of the force of the moving part is equal to the inertial force of the moving part so that $F_{\mathrm{F}}=F_{\text {mass }}=M a$. According to Fujii [12] if the force in the linear bearing $F_{\text {bearing }}$ cannot be ignored then this force shown in equation 3 should be taken into account and it is made of three parts. The first part is $F_{\text {aiflow }}$ which is the frictional force inside the air film of the linear bearing due to the asymmetrical flow of the air, the second part $F_{\text {gravity }}$ is the component of the gravitational force due to the inclination of the bearing holder against the horizontal

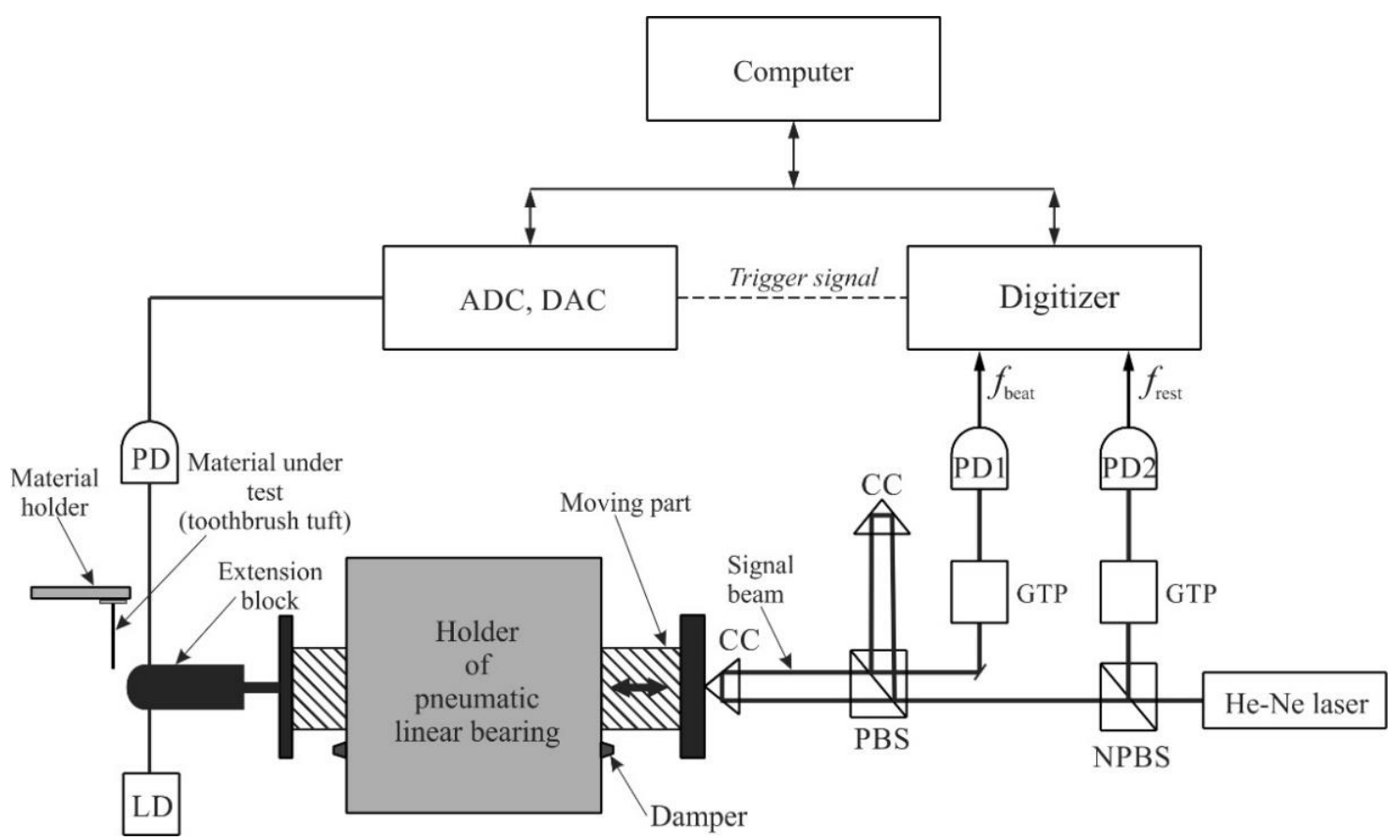

Figure 1. Schematic diagram of experimental setup. Code: $C C=$ cube corner prism, $P B S=$ polarizing beam splitter, $\mathrm{NPBS}=$ non-polarizing beam splitter, GTP $=$ Glan-Thompson prism, $\mathrm{PD}=$ photo diode, $\mathrm{LD}=$ laser diode, $\mathrm{DAC}=$ Digital-to-Analogue Converter, ADC = Analogue-to-Digital Converter. 


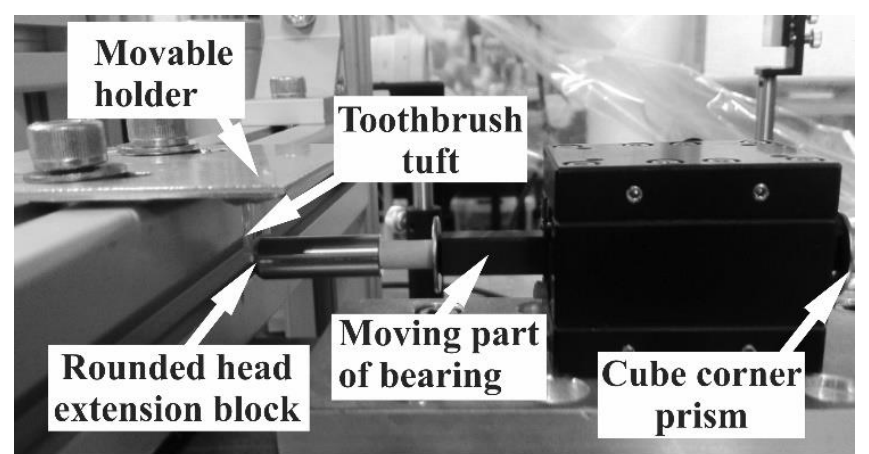

(a)

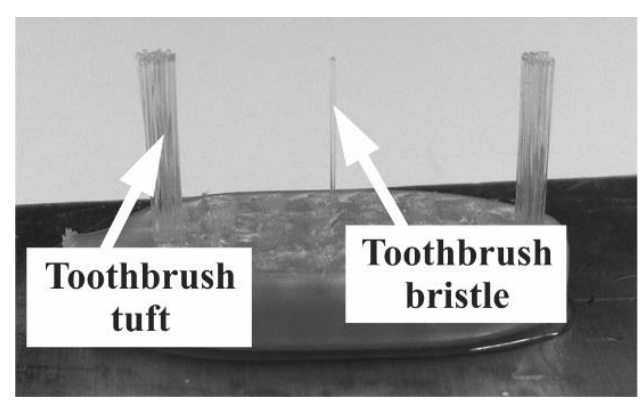

(b)

Figure 2. (a) Photograph of the test section for the friction measurement of toothbrush tuft (b) photograph of toothbrush tufts and bristle.

plane, and the third part $F_{\text {friction }}$ which is the frictional force inside the air film of the bearing due to the relative motion between the moving part and the bearing holder.

$$
F_{\text {bearing }}=F_{\text {airflow }}+F_{\text {gravity }}+F_{\text {friction }}
$$

In the measurement and the collection of data, $f_{\text {beat }}$ and $f_{\text {rest }}$ are processed through a digitizer that is connected to the computer. Then the data obtained from the computer is used to calculate the Doppler shift frequency $f_{\text {Doppler }}$ of the signal beam of the laser interferometer while the velocity of the moving part is calculated after knowing the value of $f_{\text {Doppler }}$. This expression is shown in equation 4 and equation 5 .

$$
\begin{array}{r}
f_{\text {Doppler }}=-\left(f_{\text {beat }}-f_{\text {rest }}\right) \\
v=\lambda_{\text {air }}\left(f_{\text {Doppler }}\right) / 2
\end{array}
$$

where $f_{\text {beat }}$ is the beat frequency, $f_{\text {rest }}$ is the rest frequency, and $\lambda_{\text {air }}$ is the wavelength of the signal beam under the experimental conditions. The direction of motion during the experiment as shown in Fig. 2 is towards the left for the velocity, the acceleration, and the force acting on the moving part.

The light source used in this experiment is Zeemantype two-frequency He-Ne laser. The frequency difference is measured from an interference fringe which appears at the output port of the interferometer. The value of the signal varies from $f_{\text {rest }}$ approximately $1.8 \mathrm{MHz}$ to some value depending on the velocity of the moving part. A digitizer (model 5102, National Instrument) measured and recorded the signal from PD1 and PD2. During the measurement, a sampling rate of 30 million samples per second with a record length of 5 million samples is used at 8-bit resolution, and all the settings is done using Labview software. This condition makes a sampling period of the digitizer to approximately $0.17 \mathrm{~s}$. The frequencies $f_{\text {beat }}$ and $f_{\text {rest }}$ are accurately determined using zero-crossing fitting method (ZFM) from the digitized waveforms from PD1 and PD2 [18]. During measurements, the digitizer (NI PCI5105 ) is triggered by a sharp trigger signal generated from the digital-to-analog converter (DAC). This signal is initiated by a light switch that is a combination of a laserdiode and a photodiode.

The pneumatic linear bearing is made by NSK Co., Ltd. with the model number "GLS08A50/25-2571." This bearing is attached to an adjustable tilting stage. The maximum mass that can be added to the moving part is approximately $1 \mathrm{~kg}$ according to its design specification. The stroke of its movement is approximately $25 \mathrm{~mm}$ with an air film nominal thickness of approximately $10 \mu \mathrm{m}$. The tilting angle of the upper surface of the bearing holder can be roughly adjusted horizontally using a bubble level with uncertainty of approximately $0.1 \mathrm{mrad}$. This angle equal to $0.1 \mathrm{mrad}$ makes the gravitational force $F_{\text {gravity }}$ acting on the moving part of approximately equivalent to $0.02 \mathrm{mN}$ (20 $\mu N)$.

\section{Results}

The collection of data is performed by running the rounded tip extension block over the surface of the toothbrush tuft as seen in Figs. 1 and 2. The friction force $F_{\mathrm{F}}$ resisting in the opposite direction of motion is equal to the total force produce by the moving part. The total force acting on the tuft is calculated as the product of the mass and the acceleration of the moving part. Figure 3 shows the data processing procedures on how to obtain the position, acceleration, and force. Throughout the friction measurement, there are two parameters that are precisely measured using an optical interferometer, one is the time- 


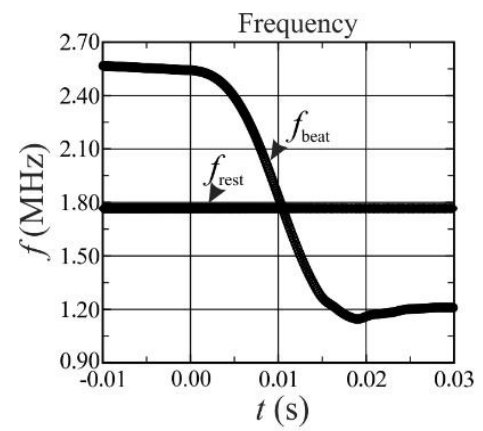

(a)

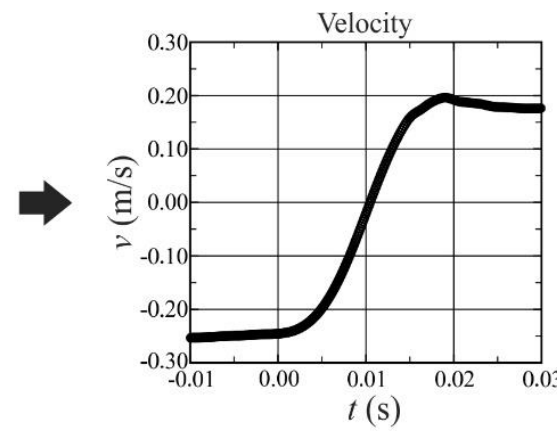

(b)

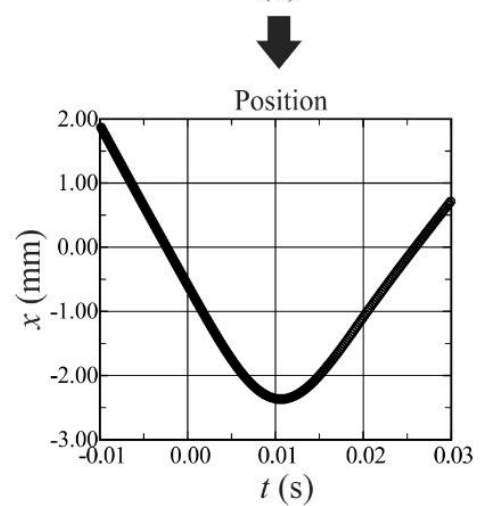

(d)

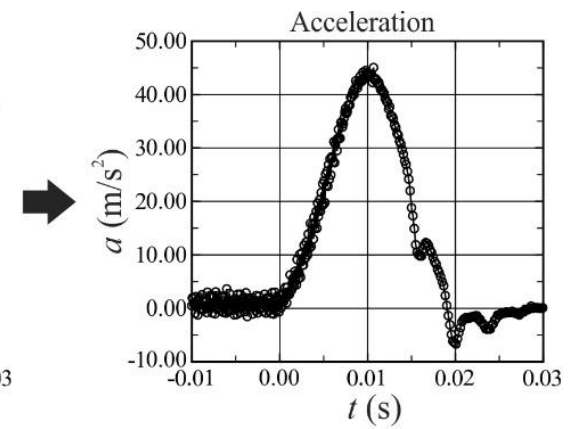

(c)

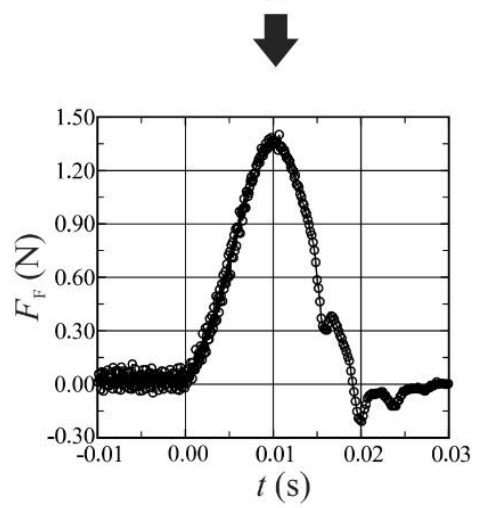

(e)

Figure 3. Data processing procedure from (a) frequency in calculating (b) velocity, (c) position, (d) acceleration, and (e) force.

varying beat frequency $f_{\text {beat }}$ and the other is the rest frequency $f_{\text {rest }}$.

The Doppler shift frequency which is needed for the calculation of velocity is measured as the difference between the beat frequency $f_{\text {beat }}$ and the rest frequency $f_{\text {rest }}$. The position and the acceleration is calculated after the calculation of velocity from the measured time-varying Doppler shift frequency. The calculation is performed by numerical integration and differentiation of the velocity. Finally, the time-varying force is calculated using the product of the moving mass and the time-varying acceleration.

Figure 3 (e) shows the change of the friction force acting on the moving mass against time. From this figure, the maximum value of the friction force $F_{\mathrm{F}, \max }$ is approximately $1.4 \mathrm{~N}$. It is also visible from this figure that there is a sudden change of shape of the curve from the time $15.16 \mathrm{~ms}$ to $16.46 \mathrm{~ms}$ and from the time $19.19 \mathrm{~ms}$ to 20.57 $\mathrm{ms}$. The cause of these changes are due to the interaction between the individual bristles composing the tuft and the surface of the extension block. As the extension block moves back to its original position some of the bristles tend to adhere each other and to the extension block surface creating a sudden increase of friction force that resist the moving part this can be seen in Fig. 2(a). This observation is supported by Fig. 4 in the position $-19.97 \mathrm{~mm}$ to -19.70 $\mathrm{mm}$ a bulge shaped can be seen in the plot of friction force against position. The negative friction force that is visible from Figs. 3 (e) and 4 indicates that the bristles provides additional force from its stored energy after experiencing elastic deformation.

Figure 4 shows that hysteresis occurs during the brushing process. But since the area is so narrow, it means that the material under test dissipated energy at a very small amount. This dissipated energy is due to internal friction of the material. Figures 3 and 4 also showed that noise in

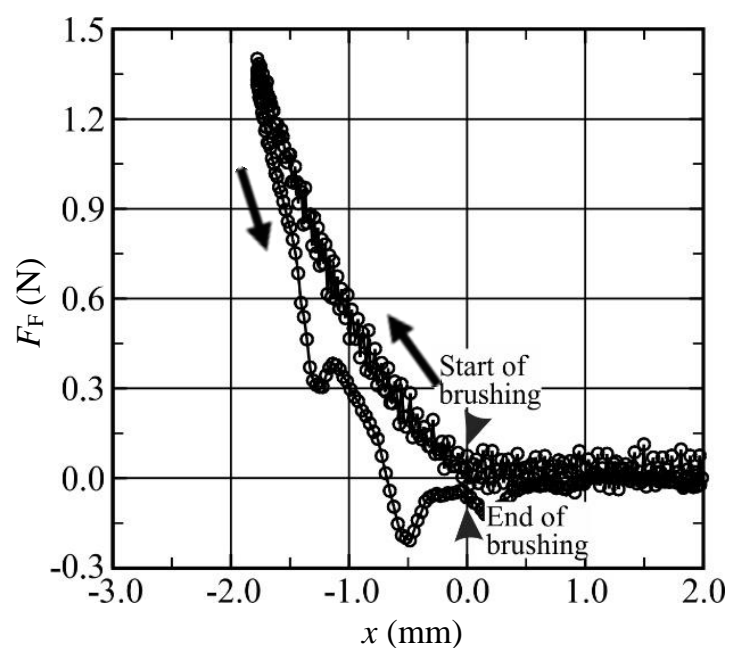

Figure 4. Change in friction force $F_{\mathrm{F}}$ with position. 
forms of friction is highly visible during the forward tapping when the moving part was given an initial velocity. However, this noise disappears quickly when the moving part attained a perfect balance in the maximum position $20.37 \mathrm{~mm}$ to the left. This is when the velocity of the moving part becomes zero. At this time, there is a reaction force coming from the damper that pushes the moving part backward after it stops. This reaction force is perfectly horizontal so that when it moves back the noise disappear.

Figure 5 shows the curve fitted equation of force against position as it brushes forward and backward from the toothbrush tuft for about $23 \mathrm{~ms}$. From this figure it is obvious that the dynamic friction force $F_{\mathrm{F}}$ is a second-order function of position with an R squared value of $99.27 \%$ for Fig. 5(a) and $97.95 \%$ for Fig. (b). It means its stiffness do not follow the hooks' law where the force is a linear function of position.

Figure 6 shows the change of the friction force with the velocity wherein the maximum friction force is attained when the velocity of the moving part becomes zero. At this time, the moving part stops and starts to reverse its direction of motion from left to right. All of this happens when the moving part reaches the maximum position. Figure 7 shows the change of velocity with position. In this figure, the velocity changes relatively before and after brushing. This change means that during the process of moving backward, the moving part already loses some of its kinetic energy due to brushing contact over the surface of the tuft.

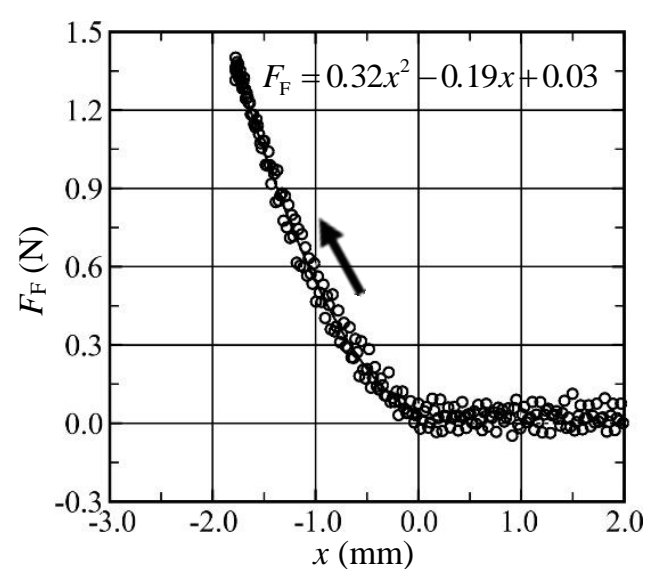

(a)

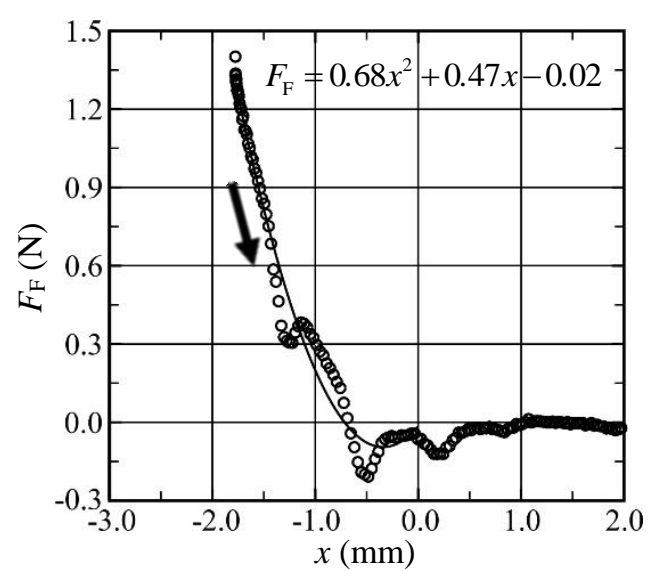

(b)

Figure 5. Curve fitted friction force $F_{\mathrm{F}}$ against position during (a) forward and (b) backward sliding motion.

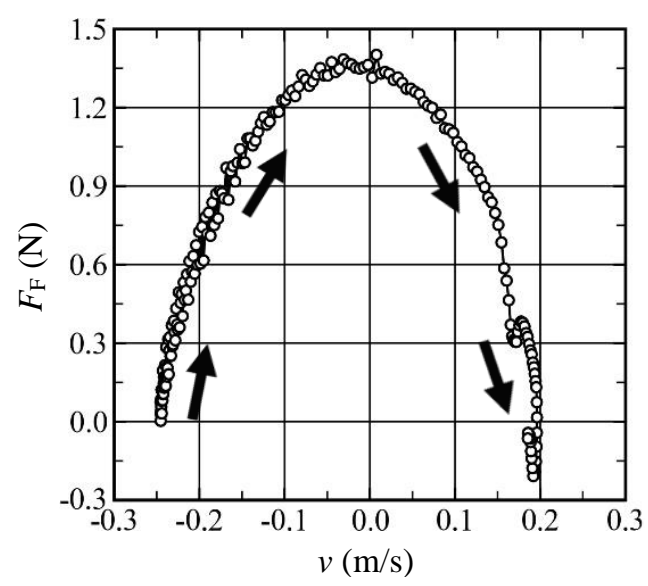

Figure 6. Change of friction force $F_{\mathrm{F}}$ with velocity.

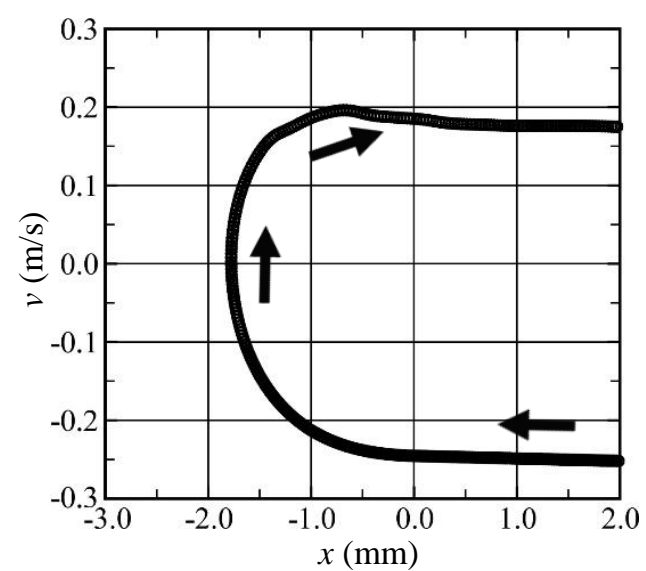

Figure 7. Change of velocity with position. 


\section{Discussion}

The Levitation Mass Method (LMM) was adopted in this experiment. During the measurement and calculation of the dynamic friction force $F_{\mathrm{F}}$, the bearing friction $F_{\mathrm{b}}$ force was neglected. This is because its average value is less than $0.5 \%$ of the maximum value of the friction force $F_{\mathrm{F}, \max }$ which is approximately equal to $1.40 \mathrm{~N}$.

During the first part of the trip when the extension block brushes over the tuft, the noise due to friction is visible. This noise can be seen in Figs. 4-8. The cause of this noise is due to the unbalance tapping of the moving part when giving an initial velocity. Using the available data from the position against friction force, the stiffness can be calculated. Although initially, the friction force against position is fitted with a second-order function. Linear fitting is more convenient to approximate its average stiffness value during forward and backward stroke. Using linear fitting the stiffness is approximately $0.83 \mathrm{~N} / \mathrm{mm}$ with an R squared value of $88 \%$.

\section{Conclusion}

In this paper, the Levitation Mass Method (LMM) was successfully applied in the measurement of dynamic friction and stiffness of the toothbrush tuft. During the experiment, the moving part with mass $M$ is levitated using pneumatic linear bearing to realize linear motion with sufficiently small friction. The position, the acceleration and the inertial force of the mass are calculated from the velocity using numerical differentiation and integration.

The success of this experiment will lead to the measurement of the coefficient of friction of the toothbrush tuft and the toothbrush itself which will give vital information in concluding efficacy of toothbrushing in relation to the friction force applied during brushing of teeth.

\section{Acknowledgement}

This work was supported in part by a research-aid fund of the NSK Foundation for the Advancement of Mechatronics (NSK-FAM) and the Grant-in-Aid for Scientific Research (B) 24360156 (KAKENHI 24360156).

\section{References}

(1) Catherine Penick, "Power toothbrushes: a critical review", Int J Dent Hyg., 2(1):40-44, 2004
(2) Roger Lewis, Rob S. Dwyer-Joyce, and Matthew J. Pickles, "Interaction between toothbrushes and toothpaste abrasive particles in simulated tooth cleaning", Wear, 257, 368-376, 2004

(3) A. Frandsen, "Mechanical oral hygiene practices: state-of-the science review", Dental plaque control measures and oral hygiene. Oxford, 93-116, 1986

(4) M. Hotta, T. Yoshida, I. Sekine, S. Imada, A. Sano, "Evaluation of tapered-end toothbrushes regarding subgingival access efficacy", J Clin Dent., 8(6):15658,1997

(5) Samuel L. Yankell, Xiuren Shi, Robert C. Emling, R. Bucker, and S. Loudin, "Laboratory evaluation of two bi-level toothbrush products for subgingival access and gingival margin cleaning”, J Clin Dent, 11(1):20-23, 2000

(6) T. Arai, Kinoshita S., "A comparison of plaque removal by different toothbrushes and toothbrushing methods", Bull Tokyo Med Dent Univ, 24(2):177-88. 1977.

(7) Andrew L. Beke, "Functional Stiffness Characteristics of Toothbrush Bristles", J Dent Res , 46: 666-671, July 1967

(8) G. A. Fridus Van der Weijden, M. F. Timmerman, E. Reijerse, CM Snoek, U. Van der Velden, "Toothbrushing force in relation to plaque removal", J Clin Periodontol, 23(8):724-29, 1996

(9) G. A. Fridus Van der Weijden , M. F. Timmerman, MM. Danser , U. Van der Velden, "Relationship between the plaque removal efficacy of a manual toothbrush and brushing force", J Clin Periodontol, 25(5): 413-16.a, 1998

(10) Yusaku Fujii, "Measurement of Force Acting on a Moving Part of a Pneumatic Linear Bearing", Review of Scientific Instruments 74:3137-3141 2003.

(11) Yusaku Fujii, "Measurement of Impulse Response of Force Transducers', Review of Scientific Instruments 72:3108-3111, 2001

(12) Yusaku Fujii, "Microforce materials tester", Review of Scientific Instruments 76, 0651112005

(13) Yusaku Fujii, "Optical method for evaluating material friction”, Meas. Sci. Technol. 15, 1971-1976 2004

(14) Yusaku Fujii, "Method for measuring transient friction coefficients for rubber wiper blades on glass surface”, Tribology International 41, 17-23, 2008

(15) Yusaku Fujii, "Possible application of mass levitation to force measurement", Metrologia, Vol.38, No.1, pp.83-84, 2001

(16) Yusaku Fujii, "Microforce materials tester based on the levitation mass method", Meas. Sci. Technol., Vol.18, No.6, pp.1678-1682, 2007 
(17) M.A. Bueno, R. Bocquet, M. Tourlonias, R.M. Rossi, S. Derler, "Study of friction mechanism of hairy textile fabrics", Wear 303, 343-353, 2013

(18) Yusaku Fujii. and Hessling J., "Experimental Techniques", 33 64-69, 2009 